\title{
Oil production predicting with modified BP neural network method
}

\author{
Haohan Liu, Wei Li, Songlin Zhang \\ Sichuan College of Architectural Technology \\ Deyang, China, 618000 \\ e-mail:tsinghua616@163.com
}

\begin{abstract}
Feasibility of oil production predicting results influence the annual planning and long-term field development plan of oil field, so the selection of predicting models plays a core role. In this paper, a common and useful model is introduced, it is,the neural network model. By using this model to predict the oil production in DAQ oilfield in China, advantages and disadvantages of the model has been discussed. The predicting results show: the fitting accuracy by the neural network model is high, and the prediction error is smaller than $10 \%$, so neural network model can be used to short-term forecast of oil production, after changing the weighting value in training, we can also improve the predicting accuracy, however, this process takes much time. Next, our team will try to develop new theory to shorten the training time.
\end{abstract}

Keywords-Oil field;oil production; neural network; predicting accuracy

\section{INTRODUCTION}

Oil production prediction is very important in the oilfield development, study on oil production predicting method is a key topic of petroleum science. At present, there are four kinds of predicting methods ${ }^{[1-6]}$ with physical meanings: Empirical formula such as Arps method, Hubbert model, water-flood decline curve method; Hydrodynamic model based on fluid mechanics model; Material balance equation model; Numerical reservoir simulation model. Besides the above mentioned four methods, there is a typical type of prediction model related to modern optimization, this model type is composed of $\mathrm{GM}(1,1)$ model, neural network model, support vector machine model, etc. Oil field development system is a complex multi variables non-linear dynamical systems, different predicting model has different characteristics like predicting accuracy.Neural network model is an effective method to solve multi nonlinear mapping problem. At present it is used in many disciplines, even in the oil production prediction. In this paper, neural network model is used to predict the oil production of XINJIANG oil field, and good predicting results have been achieved..

\section{NEURAL NETWORK PREDICTING MODEL}

At present, in the application of artificial neural networks (ANN) ${ }^{[7,8]}$, most of them are back propagation (BP) ANN and their variations. It has been proved that BP neural network can approximate any multivariate continuous function. Kolmogorov rule guaranteed that any continuous function or mapping can be achieved by a 3-layer ANN. 3- layer BP ANN is used to establish the ANN model with prediction function. The first layer of BP-ANN is input layer, the second layer of it is middle layer, and the third layer of it is the output layer, see figure 1.

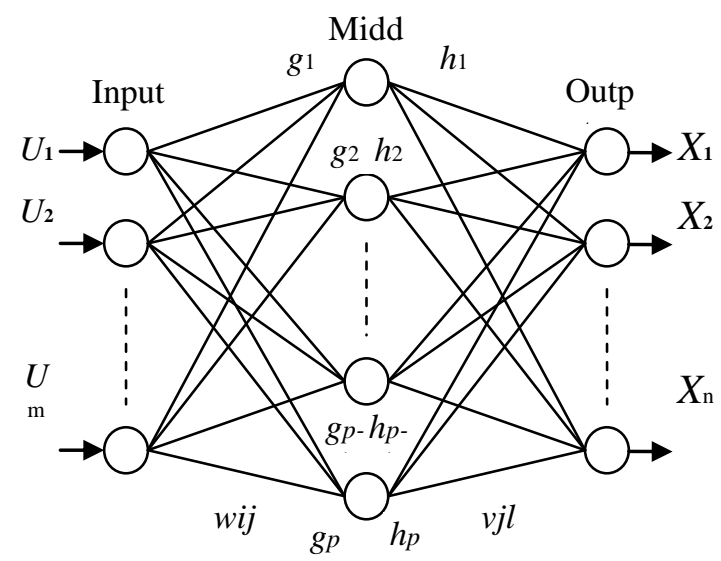

Figure1 BP neural network structure

Network simulation

Assume $N$-samples (input-output data samples) are used during the training process:

The input variables are

$U(t)=\left(u_{1}(t), u_{2}(t), \cdots, u_{m}(t)\right)^{T}$, the output variables are

$X(t)=\left(x_{1}(t), x_{2}(t), \cdots, x_{n}(t)\right)^{T}$. For the $k$-sample

$(k=1,2 \cdots N)$, let $U^{k}=\left(u_{1}{ }^{k}, u_{2}{ }^{k}, \cdots u_{s}{ }^{k}\right)$ be input mode vector; $\bar{X}^{k}=\left(\bar{x}_{1}^{k}, \bar{x}_{2}^{k} \ldots \bar{x}_{n}^{k}\right)$ be the expectation output vector; $G^{k}=\left(g_{1}{ }^{k}, g_{2}{ }^{k}, \cdots g_{p}{ }^{k}\right)$ be the middle layer input vector; $H^{k}=\left(h_{1}{ }^{k}, h_{2}{ }^{k}, \cdots h_{p}{ }^{k}\right)$ be the output unit vector of middle layer; $M^{k}=\left(m_{1}{ }^{k}, m_{2}{ }^{k}, \cdots m_{n}{ }^{k}\right)$ be the input vector of output layer; $X^{k}=\left(x_{1}^{k}, x_{2}^{k} \ldots x_{n}^{k}\right)$ be the output vector of output layer; $\left\{W_{i j}, i=1,2 \cdots m ; j=1,2 \cdots p\right\}$ be the connection weights from input layer to middle layer; $\left\{V_{j t}, j=1,2 \cdots p ; l=1,2 \cdots n\right\}$ be the connection weights from middle layer to output layer; $\left\{\theta_{j}, j=1,2 \cdots p\right\}$ be the threshold value of middle layer; $\left\{\gamma_{l}, l=1,2 \cdots n\right\}$ be the threshold value of output layer; $\eta(0<\eta<1)$ is the learning rate. 
Let the response function of ANN $\boldsymbol{f}(\boldsymbol{x})$ be Sigmoidtype function: $f(x)=\left(1+e^{-x}\right)^{-1}$

The input and output values of each neural unit satisfy the following relationship:

Middle layer:

$$
\left\{\begin{array}{l}
\text { intput }: g_{j}{ }^{k}=\sum_{i=1}^{s} w_{i j} \cdot u_{i}{ }^{k}-\theta_{j}(i=1,2 \cdots m ; j=1,2 \cdots p) \\
\text { output: } h_{j}{ }^{k}=f\left(g_{j}{ }^{k}\right)(k=1,2 \cdots N)
\end{array}\right.
$$

Output layer:

$$
\left\{\begin{array}{l}
\text { input: } m_{l}^{k}=\sum_{j=1}^{p} v_{j l} \cdot h_{j}^{k}-\gamma_{l} \quad(l=1,2 \ldots n) \\
\text { output: } x_{l}^{k}=f\left(m_{l}^{k}\right)
\end{array}\right.
$$

After training of $N$ samples, the network error is:

$$
E_{\text {total }}=\frac{1}{2} \sum_{k=1}^{N} \sum_{l=1}^{n}\left(\bar{x}_{l}^{k}-x_{l}^{k}\right)^{2} .
$$

Error of output layer unit:

$$
d_{l}^{k}=\left(\bar{x}_{l}^{k}-x_{l}^{k}\right) x_{l}^{k}\left(1-x_{l}^{k}\right)
$$

Error of middle layer unit:

$$
e_{j}^{k}=\left[\sum_{l=1}^{n} d_{l}^{k} \cdot v_{j l}\right] h_{j}^{k}\left(1-h_{j}^{k}\right)
$$

The connection weights $\left\{V_{j l}\right\}$ and the threshold value $\left\{\gamma_{t}\right\}$ can be modified by the output layer error $\left\{d_{l}^{k}\right\}$ and output value of middle layer unit $\left\{h_{j}^{k}\right\}$ :

$$
\begin{gathered}
V_{j l}(q+1)=V_{j l}(q)+\eta \sum_{k=1}^{N} d_{l}^{k} h_{j}^{k} \\
\gamma_{l}(q+1)=\gamma_{l}(q)+\eta \sum_{k=1}^{N} d_{l}^{k}
\end{gathered}
$$

The connection weights $\left\{W_{i j}\right\}$ and the threshold value $\left\{\theta_{j}\right\}$ can be modified by the middle layer error $\left\{e_{j}^{k}\right\}$ and input value of input layer $U^{k}=\left(u_{1}{ }^{k}, u_{2}{ }^{k}, \cdots u_{m}{ }^{k}\right)$ :

$$
\begin{aligned}
& W_{i j}(q+1)=W_{i j}(q)+\eta \sum_{k=1}^{N} e_{j}^{k} u_{i}^{k} \\
& \theta_{j}(q+1)=\theta_{j}(q)+\eta \sum_{k=1}^{N} e_{j}^{k}
\end{aligned}
$$

Repeat the above-mentioned learning mode, until the network converges to a given error range.

\section{EASE OF USE OF APPLICATIONS AND DISCUSSIONS}

Given the initial oil production data (from 1958 to 2012) of certain oilfield block in China, then the above-mentioned three method can be used to predict the oil production of different oilfield block(A1,A2,A3). After using the BP neural network predicting model gives figure2, figure 3 and figure4:

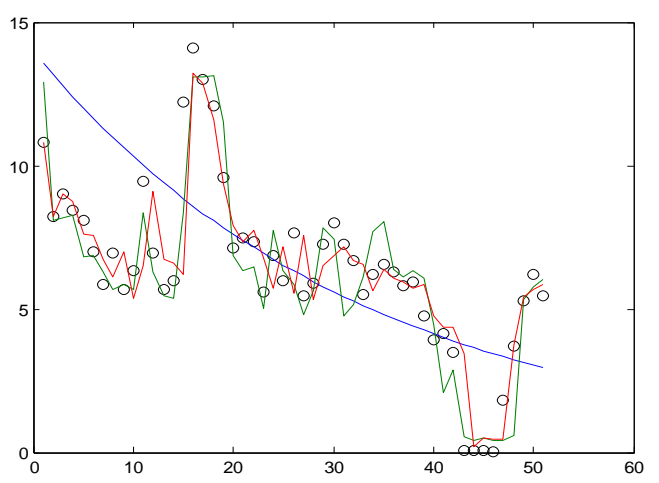

Figure2 oil production prediction value with BP neural network method in block-A1

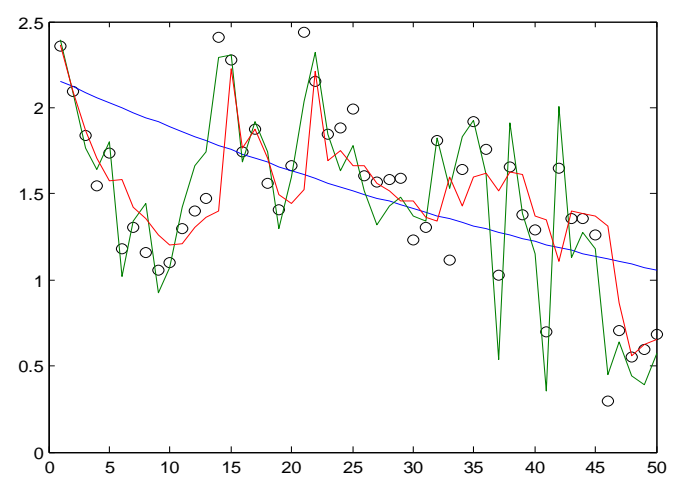

Figure 3 oil production prediction value with BP neural network method in block-A2

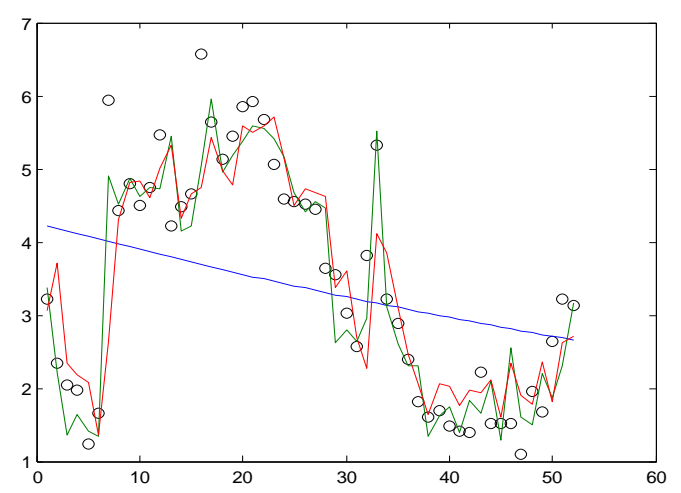

Figure4 oil production prediction value with BP neural network method in block-A3

In figure2, figure 3 and figure 4 , the black circle means the real oil production, the colored curve is the predicting 
curve with ANN model. ANN model can be used to short term prediction between 15 to 40 in figure 2 to 4 .

\section{CONCLUSIONS}

1. Prediction with ANN model model can comply with the actual oilfield production dynamics, the prediction errors of them are less than $10 \%$.

2. It is a learning type of model; much data is needed to complete the prediction, so they are only suitable for the short term prediction.

3. We can improve the predicting accuracy by modifying the weighting valuer.

\section{REFERENCES}

[1] Ji Bingyu. "Overview of Oilfield Development Indicators Forecasting Methods," Petroleum geology and oilfield development in DAQING, Vol.18, No.2, 1999, pp.19-22.
[2] Hu Jianguo, "Chen Yuanqian, Zhang Shengzong. A new model using for prediction of oil-gas field production," ACTA PETROLEI SINICA, Vol.16, No.1, 1995, pp.79-86.

[3] Chen Gan,Yang Xinling. "AN EXAMINATION INTO PREDICTION METHODS BASED ON RESERVOIR PERFORMANCE ANALYSES," CHINA OFFSHORE OIL AND GAS(GEOLOGY), Vol.16, No.4, 2002, pp. 254-259.

[4] Chen Yuanqian, Zhao Qingfei. "A Combined Method of Weibull Model and Water Drive Curve of B Type," XINJIANG PETROLEUM GEOLOGY, 2000, 21(5): 405-407.

[5] Chen Yuanqian, Hu Jianguo, Zhang Dongjie. "Deduction of Logistic model and auto regression methods," XINJIANG PETROLEUM GEOLOGY, Vol.17, No.2, 1996, pp. 150-155.

[6] Yue Ping, Chen Xiaofan, Cui Ligong, etc. "suggestion solution of Weng's model and Logistic model in production research," Journal of oil and gas technology, Vol.31, No.4, 2009, pp. 277-279.

[7] Yang Bin. "Applied neural network in oil well logging,"Beijing: Petroleum Industry Press,2005

[8] $\mathrm{Wu}$ Xingen, Ge Jiali. "oil production forecast with ANN method,"Petroleum Exploration and Development, Vol.21, No.3, 1994, pp.31-42. 